\title{
Research Square \\ Propagation of Chirped Sinh-Gaussian Beams in Uniaxial Crystals Orthogonal to the Optical Axis
}

Mert BAYRAKTAR ( $\sim$ mert.bayraktar@tai.com.tr)

Turkish Aerospace Industries https://orcid.org/0000-0002-0337-7650

\section{Research Article}

Keywords: chirped sinh-Gaussian beam, uniaxial crystal, propagation

Posted Date: June 8th, 2021

DOI: https://doi.org/10.21203/rs.3.rs-540353/v1

License: (c) (i) This work is licensed under a Creative Commons Attribution 4.0 International License.

Read Full License

Version of Record: A version of this preprint was published at Optical and Quantum Electronics on August 14th, 2021. See the published version at https://doi.org/10.1007/s11082-021-03184-4. 


\title{
Propagation of chirped sinh-Gaussian beams in uniaxial crystals orthogonal to the optical axis
}

\author{
Mert Bayraktar \\ Turkish Aerospace Industries, \\ Havacılık Bulvarı No:17, Kahramankazan 06980, Ankara, Turkey \\ Tel : 90-312-8111800, Fax : 90-312-8111425, e-mail : mert.bayraktar@tai.com.tr
}

\begin{abstract}
This article includes intensity evolutions and phase changes of chirped sinh-Gaussian(CSG) beam propagating in uniaxial crystal orthogonal to optical axis. Received field expression is calculated utilizing Huygens-Fresnel integration. We see that chirped parameter brings decentered intensity distribution. By adjusting the decay factors, higher peak intensity can be obtained at the receiver side. Broken phase at near field is corrected at phase field. Phase change and amount of circles can be controlled by decay factor and chirped parameter. We hope that our results are helpful for scientist working on optical tracking.
\end{abstract}

Keywords : chirped sinh-Gaussian beam, uniaxial crystal, propagation

\section{Introduction}

Propagation of nonconventional beams in uniaxial crystal orthogonal to optical axis is studied by scientists due to the effect of crystal on received field. Regarding with this, we show that Airyprime beam has a flat topped shape after propagation [1]. Authors investigate that extraordinary refractive index of crystal strongly influences the autofocusing of Pearcey beam [2]. Change is polarization of radially polarized Pearcey beam is discussed in [3]. Received field is obtained benefiting from convolution property of Fourier transform when Airy beam is used initially [4]. It is shown in [5] that maximum intensity of Airy-Gaussian beam propagating in uniaxial crystal is inversely proportional to ratio of extraordinary refractive index to ordinary. 
In addition to intensity, phase information of Airy Gaussian vortex beam is lost at long distance in crystal having large refractive index ratio [6]. Influence of distribution factor of AiryGaussian vortex beam is studied in nonparaxial analysis [7]. Chirped parameter of chirped Airy vortex beam controls the intensity and phase information during propagation [8]. In another nonparaxial study, authors state that chirped parameter of chirped Airy vortex beam has an effect on angular momentum at the receiver side [9]. As compared to linear and quadratic chirped parameter, Gaussian factor of chirped Airy Gaussian vortex beam effects intensity more [10]. Change in slope of radially polarized chirped Airy beam is slower in y-direction than in x-direction [11]. Initial angle of radially polarized Airy-Gaussian beams brings off-axis intensity at the receiver plane [12]. Super Lorentz-Gauss $S L G_{01}$ mode evolves into elliptic shape lying along $\mathrm{x}$-axis when ratio of refractive index is greater than one [13]. Intensity components of Hermite-Laguerre-Gaussian beam propagating in uniaxial crystal can be listed from low to high as $y, x$, and z [14]. Furthermore, inverse relation between longitudinal gradient force of rotating elliptical chirped Gaussian beam and propagation distance is mentioned in [15].

Untraditional beams which contain trigonometric functions in the source field expression take place in the literature several times. Regarding with this, we show intensity evolution of hyperbolic sinusoidal beam under strong turbulence and it is concluded as astigmatic beam protects their shape at longer distances [16]. Besides this, sinh-Gaussian vortex beam provides higher signal to noise ratio than sinh-Gaussian beam since its scintillation is less [17]. Rootmean-square width of partially coherent sinh-Gaussian beam is directly proportional with zenith angle [18]. In strongly nonlocal nonlinear media, hollow sinh-Gaussian beam has a periodic evolution in its intensity [19]. The same beam is focused onto a nano sized sphere and it is found that deeper potential than Gauss beam can be obtained [20]. In addition, circular outer rings occur during the propagation of astigmatic hyperbolic sinusoidal Gaussian beam under 
oceanic turbulence [21]. By controlling the spiral parameter, intensity of spirally polarized sinhGaussian beam in the focal region is decreased [22].

In this report, propagation properties of chirped sinh-Gaussian beam are studied in detail. We compute received field applying Huygens-Fresnel integration. Effects of crystal structure, attenuation, chirped parameter are plotted at different propagation distances. Variations in intensity and phase are investigated in detail. We hope that outcomes of this study will be used in optical applications.

\section{Derivation of received field in uniaxial crystal}

We define the source field expression of chirped sinh-Gaussian beam as

$u_{s}\left(s_{x}, s_{y}\right)=\sinh \left(a s_{x}\right) \sinh \left(b s_{y}\right) \exp \left(-\frac{s_{x}^{2}+s_{y}^{2}}{w^{2}}\right) \exp \left(\frac{c s_{x}}{w_{x}}+\frac{d s_{y}}{w_{y}}+j \beta\left(\frac{s_{x}}{w_{x}}+\frac{s_{y}}{w_{y}}\right)\right)$

where $c$ and $d$ are the decay factors, $\beta$ being the chirped parameter, $a$ and $b$ are argument of sinh function, and $w$ refers to Gaussian source size. We take relative di-electric tensor of crystal from [23] as

$$
\epsilon=\left(\begin{array}{ccc}
n_{e}^{2} & 0 & 0 \\
0 & n_{0}^{2} & 0 \\
0 & 0 & n_{0}^{2}
\end{array}\right)
$$

where $n_{e}$ and $n_{0}$ correspond to extraordinary and ordinary refractive indexes of crystal. We set $n_{0}$ to 2.62. Extraordinary refractive index, $n_{e}$,calculated as $e n_{0}$. Then, received field is in uniaxial crystal is calculated as [24]

$$
\begin{gathered}
u_{r}\left(r_{x}, r_{y}\right)=\frac{k n_{0}}{2 \pi j z} \exp \left(-j k n_{e} z\right) \\
x \int_{-\infty}^{\infty} \int_{-\infty}^{\infty} u_{s}\left(s_{x}, s_{y}\right) \exp \left(\frac{j k}{2 z n_{e}}\left[n_{0}^{2}\left(r_{x}-s_{x}\right)^{2}+n_{e}^{2}\left(r_{y}-s_{y}\right)^{2}\right]\right) d s_{x} d s_{y}
\end{gathered}
$$

Here, $k$ denote wave number and it is calculated using operation wavelength as $2 \pi / \lambda$. Operation wavelength is taken as $0.53 \mu \mathrm{m}$ in this study. Additionally, propagation distance is indicated as $Z$ and intensity and phase plots are obtained considering the multiples of Rayleigh 
distance $z_{R}=k w^{2}$. Lastly, $\left(r_{x}, r_{y}\right)$ refer to transverse receiver or observation plane coordinates. Based on calculations in [25], we evaluate received field as

$$
\begin{aligned}
& u_{r}\left(r_{x}, r_{y}\right)=-\frac{k n_{0}}{2 \pi j z} \exp \left(j k n_{e} z\right) \frac{\pi}{\sqrt{\left(\frac{j k \omega^{2} n_{0}^{2}-2 z n_{e}}{2 z n_{e} \omega^{2}}\right)\left(\frac{j k \omega^{2} n_{e}-2 z}{2 z \omega^{2}}\right)}} \\
& x \exp \left(\frac{j k n_{0}^{2}}{2 z n_{e}} r_{x}^{2}+\frac{j k n_{e}}{2 z} r_{y}{ }^{2}\right) \\
& x\left\{\begin{array}{l}
\exp \left(\frac{\left(-\frac{j k n_{0}^{2} r_{x}}{z n_{e}}+a+\frac{c}{w_{x}}+\frac{j \beta}{w_{x}}\right)^{2}}{4\left(\frac{j k \omega^{2} n_{0}^{2}-2 z n_{e}}{2 z n_{e} \omega^{2}}\right)}+\frac{\left(-\frac{j k n_{e} r_{y}}{z}+b+\frac{d}{w_{y}}+\frac{j \beta}{w_{y}}\right)^{2}}{4\left(\frac{j k \omega^{2} n_{e}-2 z}{2 z \omega^{2}}\right)}\right) \\
-\exp \left(\frac{\left(-\frac{j k n_{0}^{2} r_{x}}{z n_{e}}+a+\frac{c}{w_{x}}+\frac{j \beta}{w_{x}}\right)^{2}}{4\left(\frac{j k \omega^{2} n_{0}^{2}-2 z n_{e}}{2 z n_{e} \omega^{2}}\right)}+\frac{\left(-\frac{j k n_{e} r_{y}}{z}-b+\frac{d}{w_{y}}+\frac{j \beta}{w_{y}}\right)^{2}}{4\left(\frac{j k \omega^{2} n_{e}-2 z}{2 z \omega^{2}}\right)}\right) \\
-\exp \left(\frac{\left(-\frac{j k n_{0}^{2} r_{x}}{z n_{e}}-a+\frac{c}{w_{x}}+\frac{j \beta}{w_{x}}\right)^{2}}{4\left(\frac{j k \omega^{2} n_{0}^{2}-2 z n_{e}}{2 z n_{e} \omega^{2}}\right)}+\frac{\left(-\frac{j k n_{e} r_{y}}{z}+b+\frac{d}{w_{y}}+\frac{j \beta}{w_{y}}\right)^{2}}{4\left(\frac{j k \omega^{2} n_{e}-2 z}{2 z \omega^{2}}\right)}\right) \\
+\exp \left(\frac{\left(-\frac{j k n_{0}^{2} r_{x}}{z n_{e}}-a+\frac{c}{w_{x}}+\frac{j \beta}{w_{x}}\right)^{2}}{4\left(\frac{j k \omega^{2} n_{0}^{2}-2 z n_{e}}{2 z n_{e} \omega^{2}}\right)}+\frac{\left(-\frac{j k n_{e} r_{y}}{z}-b+\frac{d}{w_{y}}+\frac{j \beta}{w_{y}}\right)^{2}}{4\left(\frac{j k \omega^{2} n_{e}-2 z}{2 z \omega^{2}}\right)}\right)
\end{array}\right\}
\end{aligned}
$$

\section{Results and discussions}

In this part of the manuscript, comments on numerical plots for derivations above. In order to see the influence of decay factors and chirped parameter, we set $a=b=1$. Firstly, we see from Fig. 1 that CSG beam with $a=b=1$ and $\beta=0.6$ has an off-axis elliptical distribution. Amount of shifting along y-axis is greater than the one along $\mathrm{x}$-axis. Additionally, center of elliptic intensity shifts more and beam enlarges as propagation distance increases. In Fig. 2, chirped parameter is selected as 0.1 . We investigate that shift in center of elliptic intensity is 
less as compared to Fig. 1. Fig. 3 and 4 involve received intensity plots for the beams in Fig. 1 and 2 respectively when $e=1.7$. Beam size in Figs. 3 and 4 is less than the ones in Figs. 1 and 2 in order. Moreover, peak intensity in related plots is higher than in Figs. 1 and 2. Fig. 5 is plotted to see the effect of $c$. In the comparison of Figs. 3 and 5, we investigate that peak intensity raises significantly when $c$ increases. Furthermore, similar decentered positions are observed. By comparing Figs. 3 and 6, we see that approximately 100 times higher peak intensity can be measured at longer propagation distances. Beam still protects its decentered position. Significant amount of intensity decay is observed for all intensity plots when propagation distance reaches to $2 z_{R}$.

In other point of view, we analyze the phase variations during propagation. CSG beam with $c=d=1$ and $\beta=0.6$ has a circular non-uniform and off-axis phase distribution at near field in crystal with $e=0.7$. When $z=z_{R}$, broken in phase is recovered and phase distribution becomes uniform by locating on-axis. Phase circles place compactly when we go through the edge of the observation plane. When chirped parameter is set as 0.1 as it is in Fig. 2, strict phase break is observed along y axis at near field. Beam recovers itself at longer distances and becomes smooth. As compared to Fig. 7, we change the crystal setting as $e=1.7$ in Fig. 9 . We see that number of circles in the observation plane is inversely proportional to refractive index ratios. Similar with higher ratio, phase is broken at close distance and then it recovers itself in longer distances. Comparing Fig. 8 and 10, we see that effect of strict phase break gets better at $z=2 z_{R}$. Similar with the previous investigation, circles are located frequently in this setting of crystal. In the comparison of Fig. 9 and 11, we investigate that raise in parameter $c$ brings us a slight phase change at near field but phase seems still close to uniform. In other words, phase is not affected by the variations in parameter $c$. On the other hand, off-axis localization of phase distribution along $\mathrm{x}$-axis attracts the attention after $z=2 z_{R}$. Lastly, change in parameter $d$ change the origin of the phase circles along y-axis. In proportion to 
parameter $c$, important phase break is observed at near field and beam enhances itself at longer distances.

\section{Conclusion}

We derive the received field expression of CSG beam propagating in uniaxial crystal by solving the Huygens-Fresnel integral. We investigate that decentered beam can be obtained by adjusting chirped parameter. Peak intensity is strongly dependent on the decay factor. Higher intensity is measured when decay factor is increased. Chirped parameter shifts phase distribution to off-axis position. While beam has non-uniform phase at close distance, uniform phase distribution is observed at longer distances. Decay factors are also effects the center of the phase distribution. We anticipate that these results will be beneficial in optical applications.

\section{Declerataions}

The authors declare no conflicts of interests. 


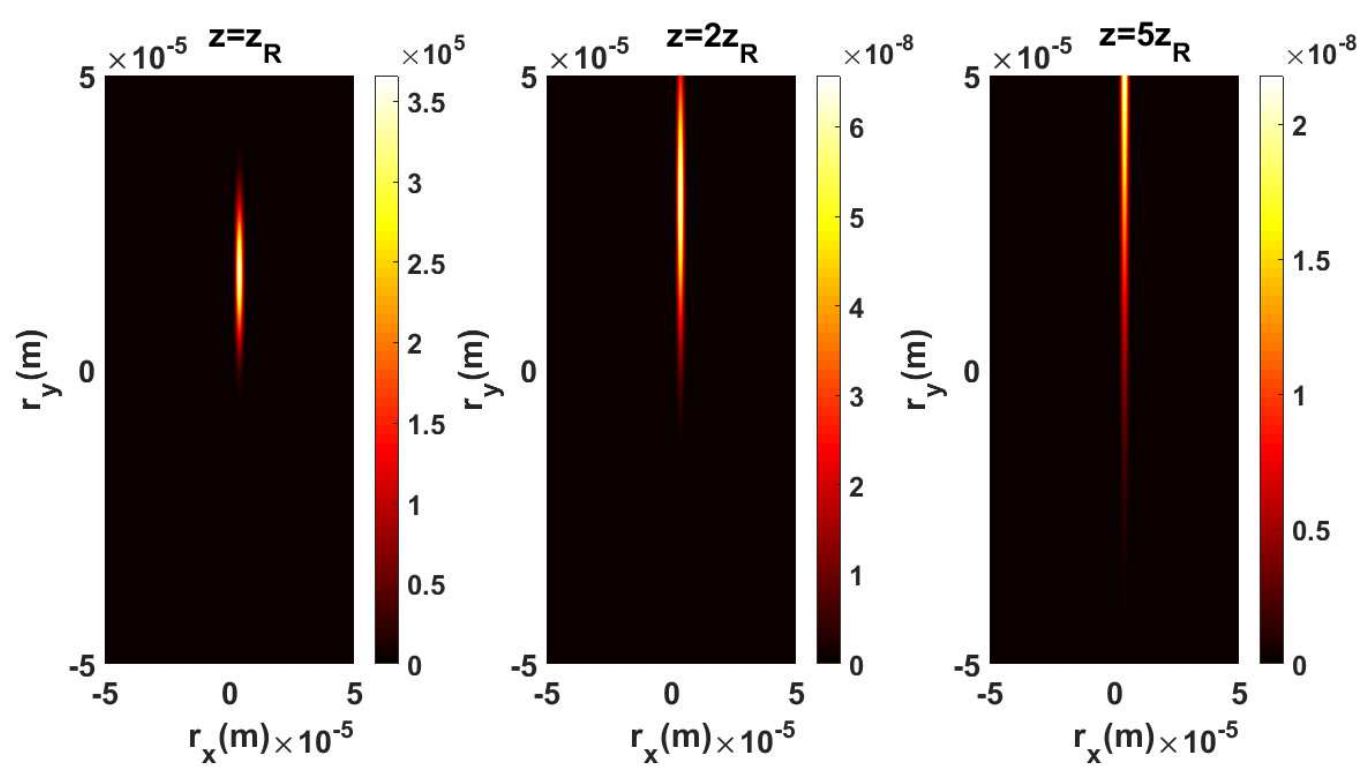

Figure 1. Intensity distribution of chirped $\sinh -$ Gaussian beam having $\boldsymbol{c}=\boldsymbol{d}=\mathbf{1}$ and $\boldsymbol{\beta}=\mathbf{0 . 6}$ in crystal with $\boldsymbol{e}=\mathbf{0 . 7}$.

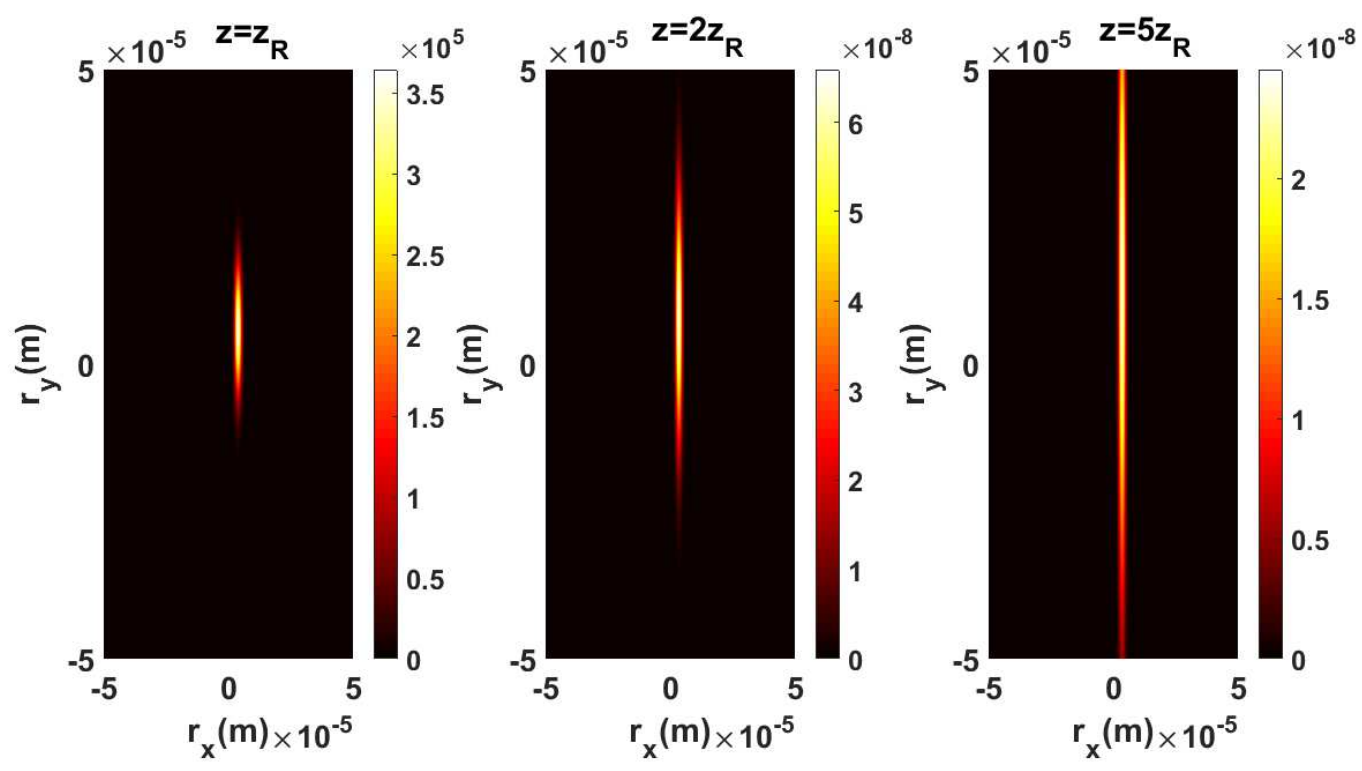

Figure 2. Intensity distribution of chirped $\sinh -$ Gaussian beam having $\boldsymbol{c}=\boldsymbol{d}=\mathbf{1}$ and $\boldsymbol{\beta}=\mathbf{0 . 1}$ in crystal with $\boldsymbol{e}=\mathbf{0 . 7}$. 


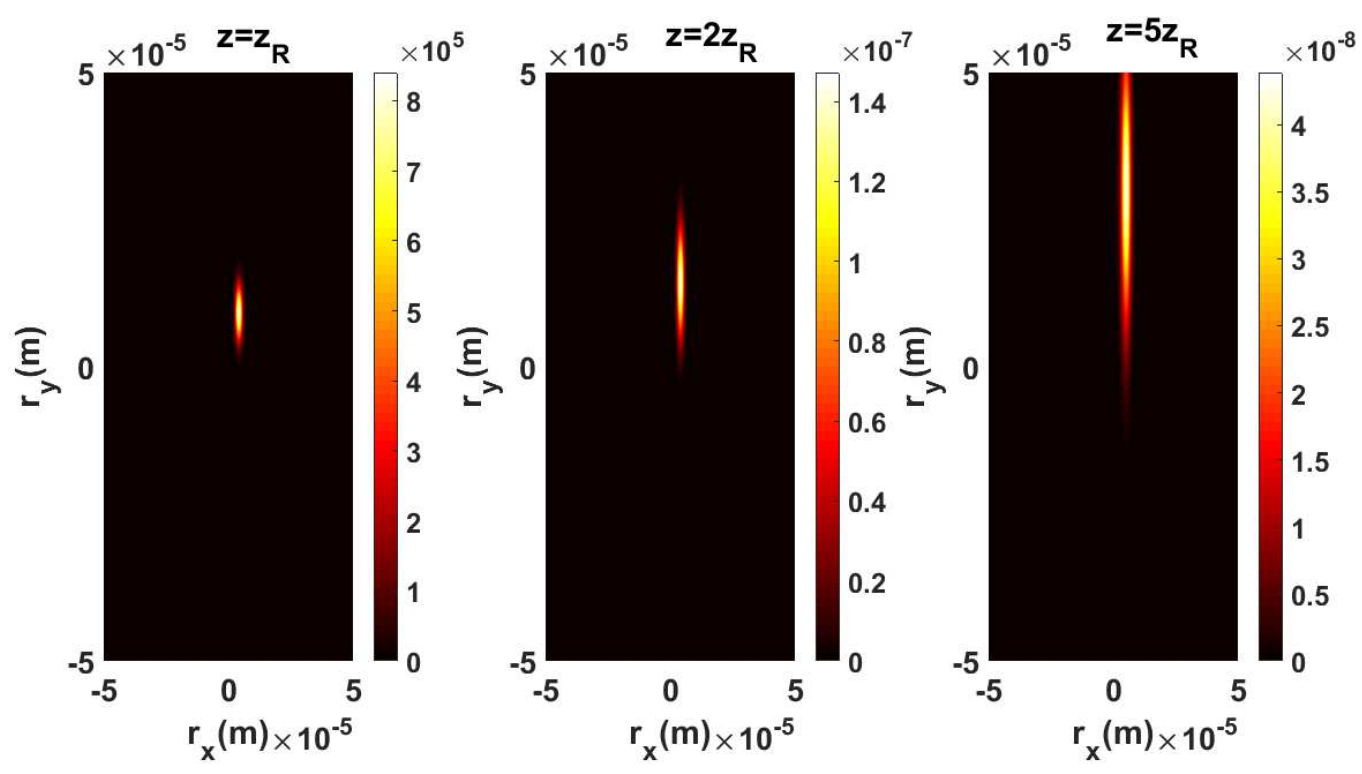

Figure 3. Intensity distribution of chirped $\sinh -$ Gaussian beam having $\boldsymbol{c}=\boldsymbol{d}=\mathbf{1}$ and $\boldsymbol{\beta}=\mathbf{0 . 6}$ in crystal with $\boldsymbol{e}=\mathbf{1} .7$.

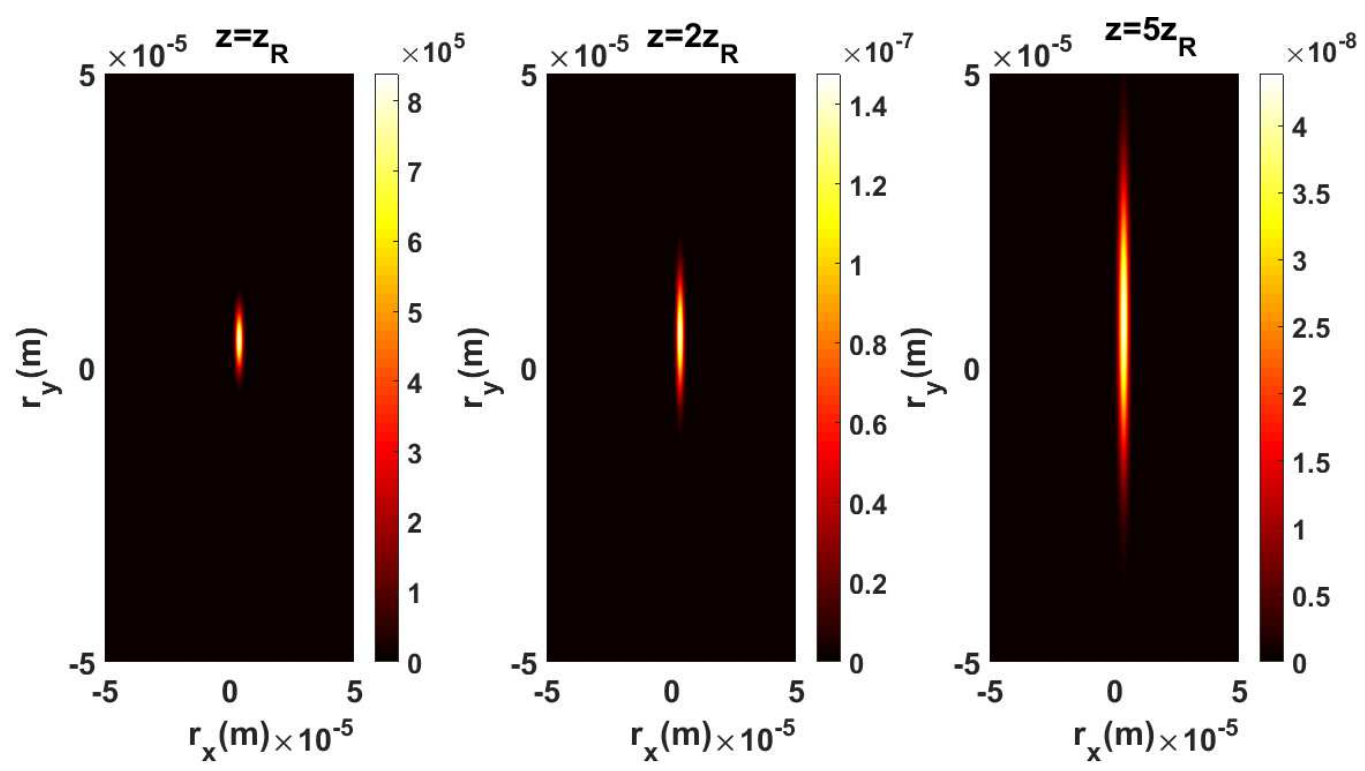

Figure 4. Intensity distribution of chirped sinh-Gaussian beam having $\boldsymbol{c}=\boldsymbol{d}=\mathbf{1}$ and $\boldsymbol{\beta}=\mathbf{0} . \mathbf{1}$ in crystal with $\boldsymbol{e}=\mathbf{1 . 7}$. 


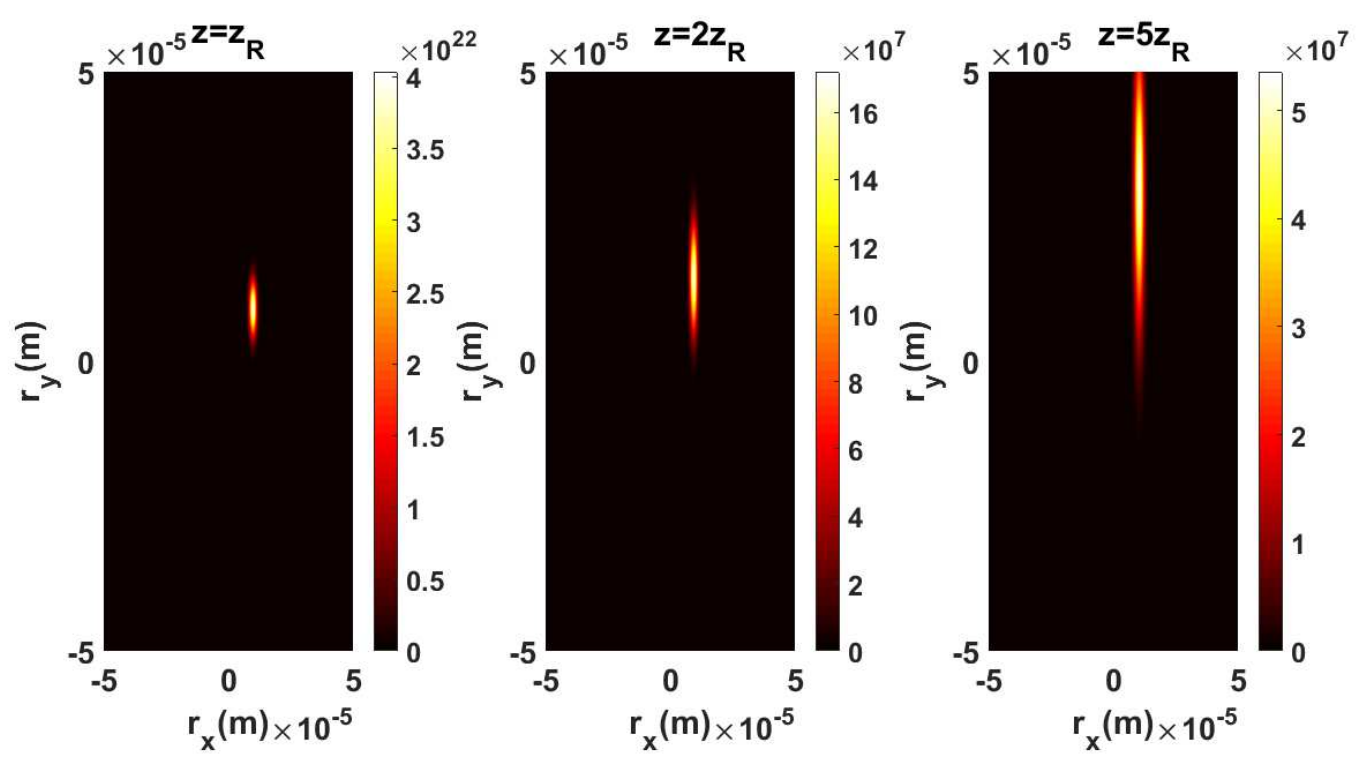

Figure 5. Intensity distribution of chirped sinh-Gaussian beam having $\boldsymbol{c}=\mathbf{3}, \boldsymbol{d}=\mathbf{1}$ and $\boldsymbol{\beta}=$ $\mathbf{0 . 6}$ in crystal with $\boldsymbol{e}=\mathbf{1 . 7}$.

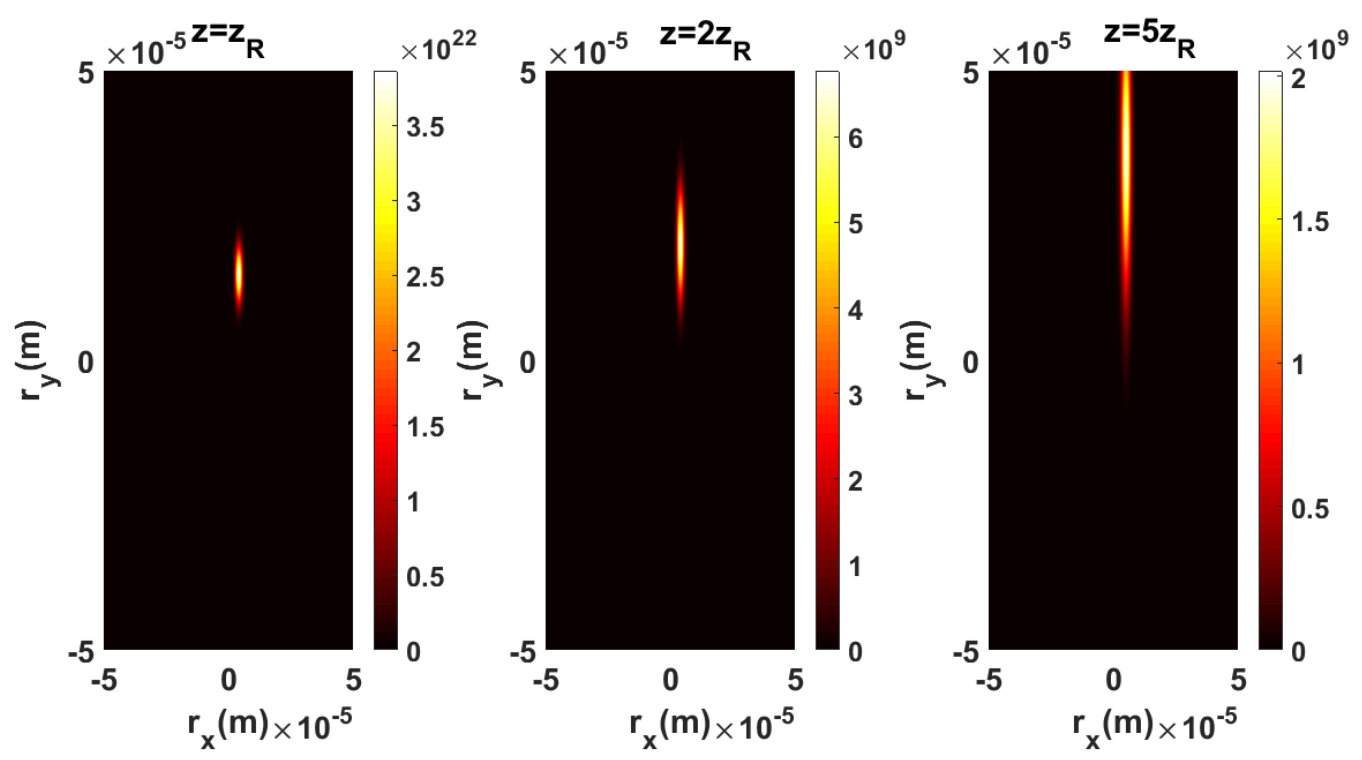

Figure 6. Intensity distribution of chirped sinh-Gaussian beam having $\boldsymbol{c}=\mathbf{1}, \boldsymbol{d}=\mathbf{3}$ and $\boldsymbol{\beta}=$ $\mathbf{0 . 6}$ in crystal with $\boldsymbol{e}=\mathbf{1 . 7}$. 

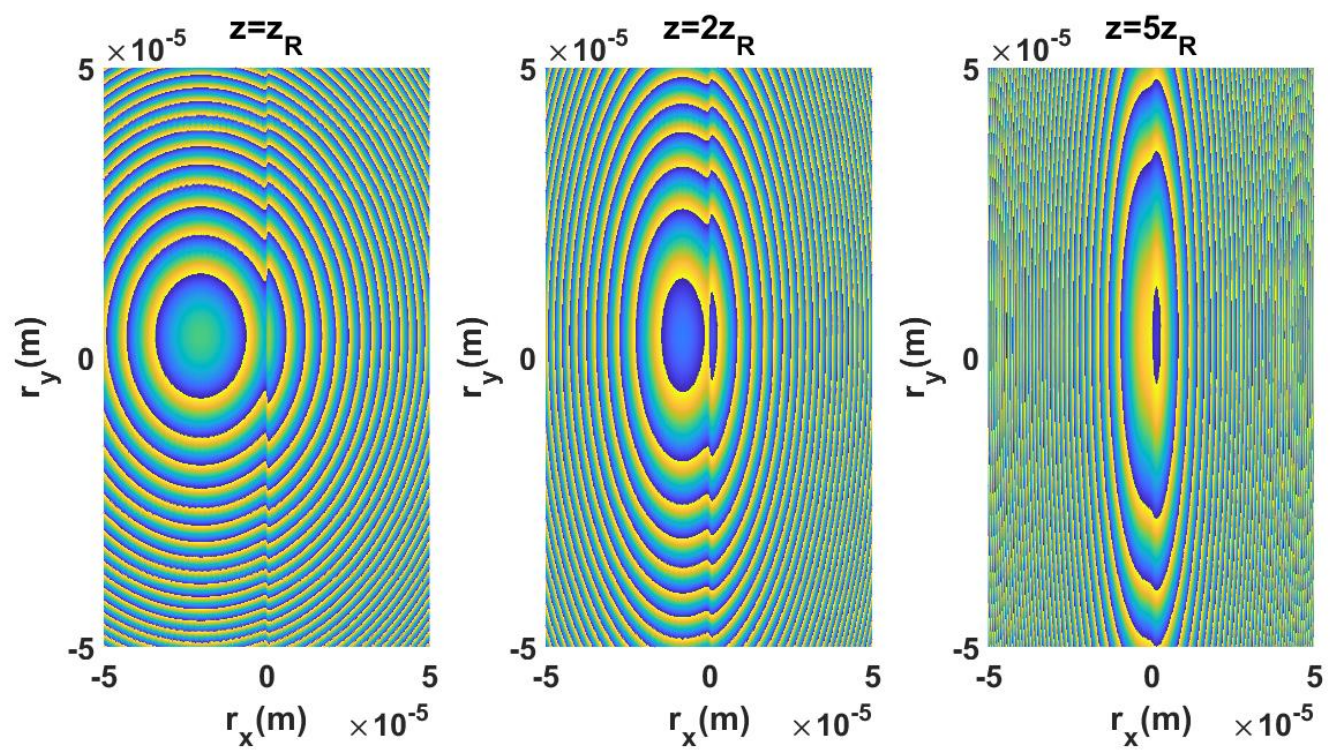

Figure 7. Phase distribution of chirped sinh-Gaussian beam having $\boldsymbol{c}=\boldsymbol{d}=\mathbf{1}$ and $\boldsymbol{\beta}=\mathbf{0 . 6}$ in crystal with $\boldsymbol{e}=\mathbf{0 . 7}$.
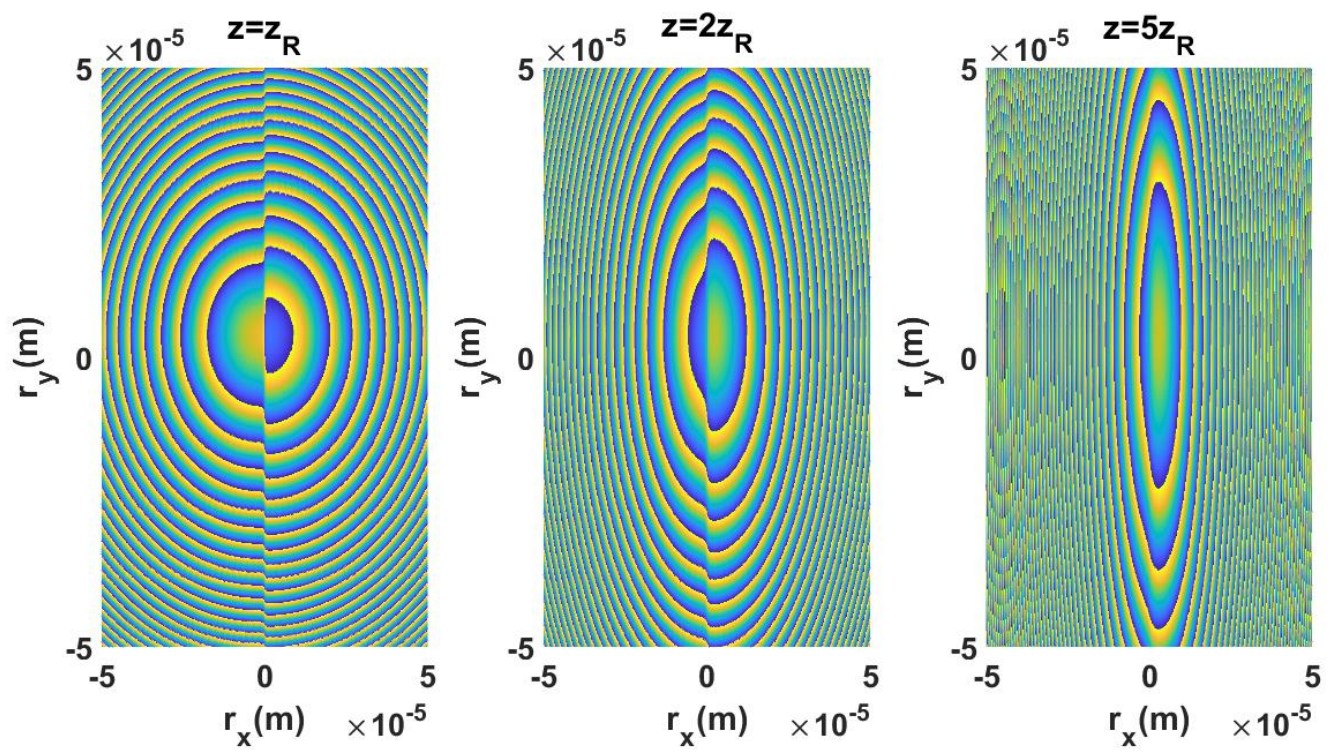

Figure 8. Phase distribution of chirped sinh-Gaussian beam having $\boldsymbol{c}=\boldsymbol{d}=\mathbf{1}$ and $\boldsymbol{\beta}=\mathbf{0 . 1}$ in crystal with $\boldsymbol{e}=\mathbf{0 . 7}$. 

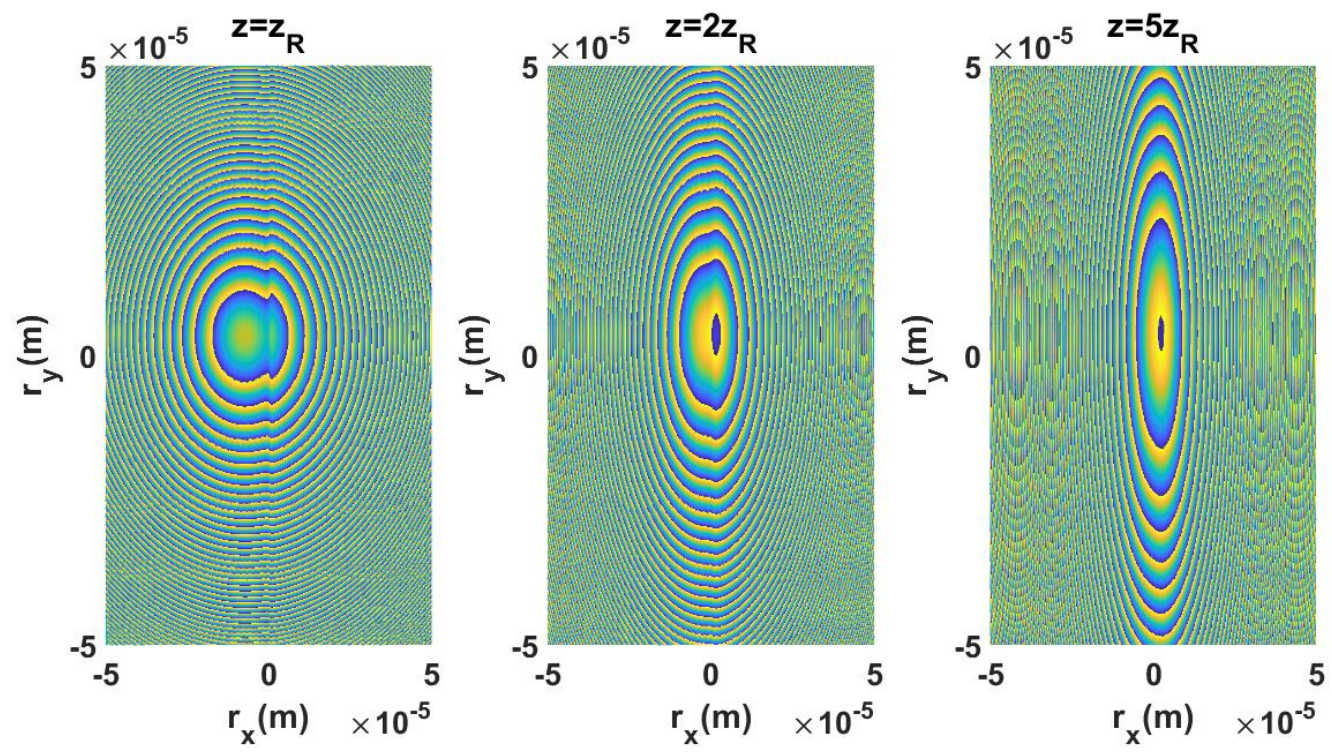

Figure 9. Phase distribution of chirped sinh-Gaussian beam having $\boldsymbol{c}=\boldsymbol{d}=\mathbf{1}$ and $\boldsymbol{\beta}=\mathbf{0 . 6}$ in crystal with $\boldsymbol{e}=\mathbf{1 . 7}$.
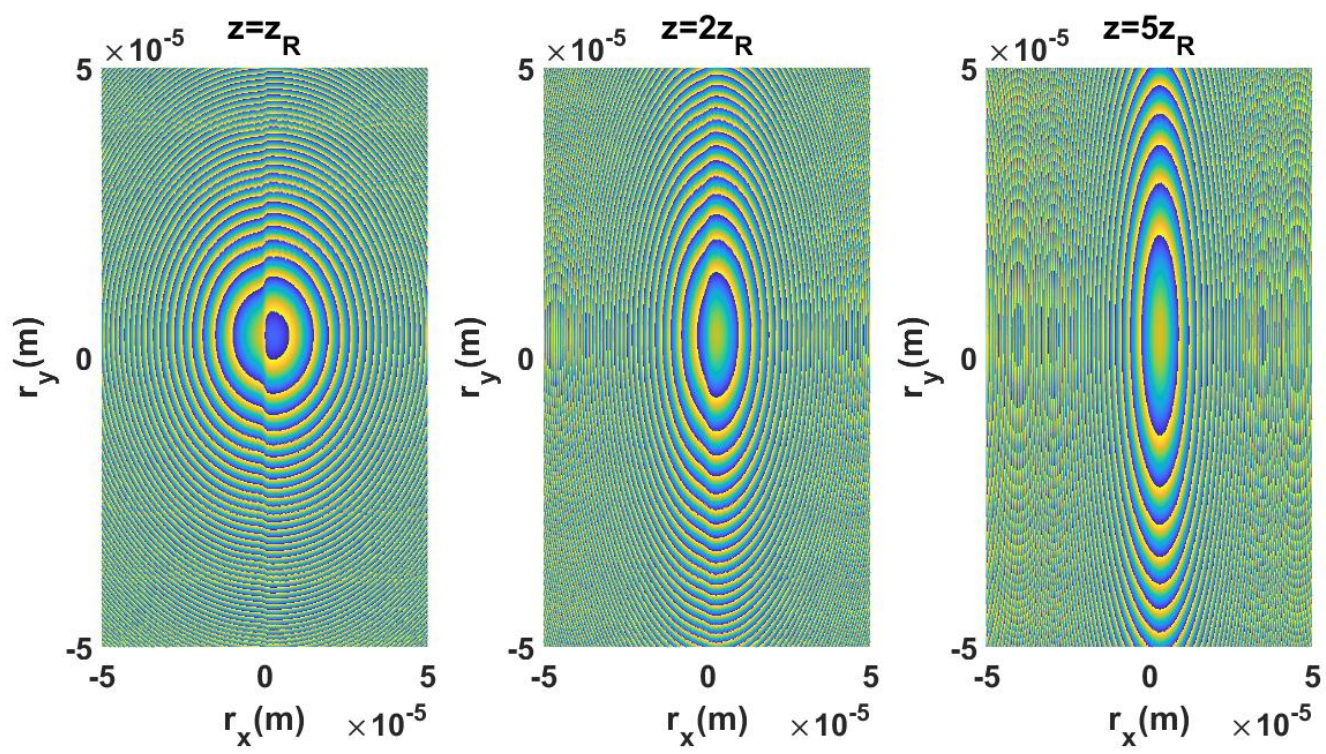

Figure 10. Phase distribution of chirped sinh-Gaussian beam having $\boldsymbol{c}=\boldsymbol{d}=\mathbf{1}$ and $\boldsymbol{\beta}=\mathbf{0} . \mathbf{1}$ in crystal with $\boldsymbol{e}=\mathbf{1 . 7}$. 

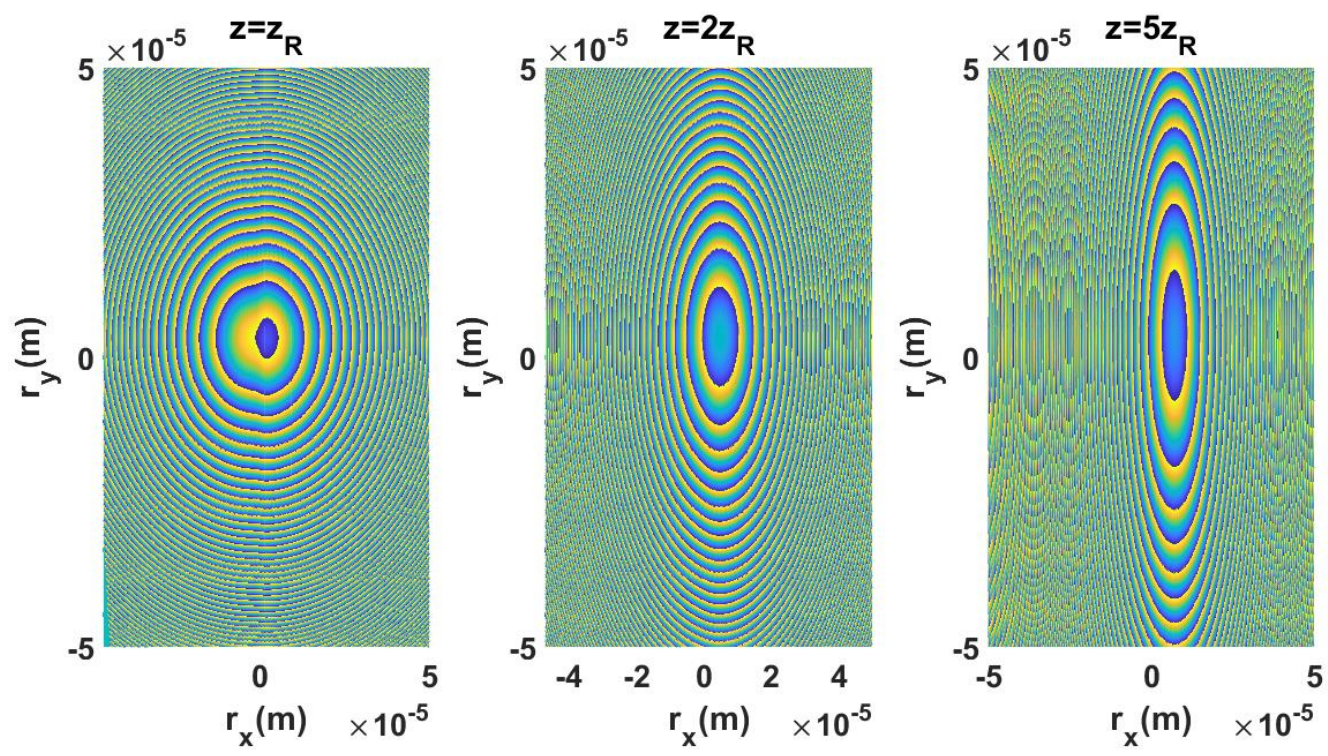

Figure 11. Phase distribution of chirped sinh-Gaussian beam having $\boldsymbol{c}=\mathbf{3}, \boldsymbol{d}=\mathbf{1}$ and $\boldsymbol{\beta}=$ 0. 6 in crystal with $\boldsymbol{e}=\mathbf{1 . 7}$.
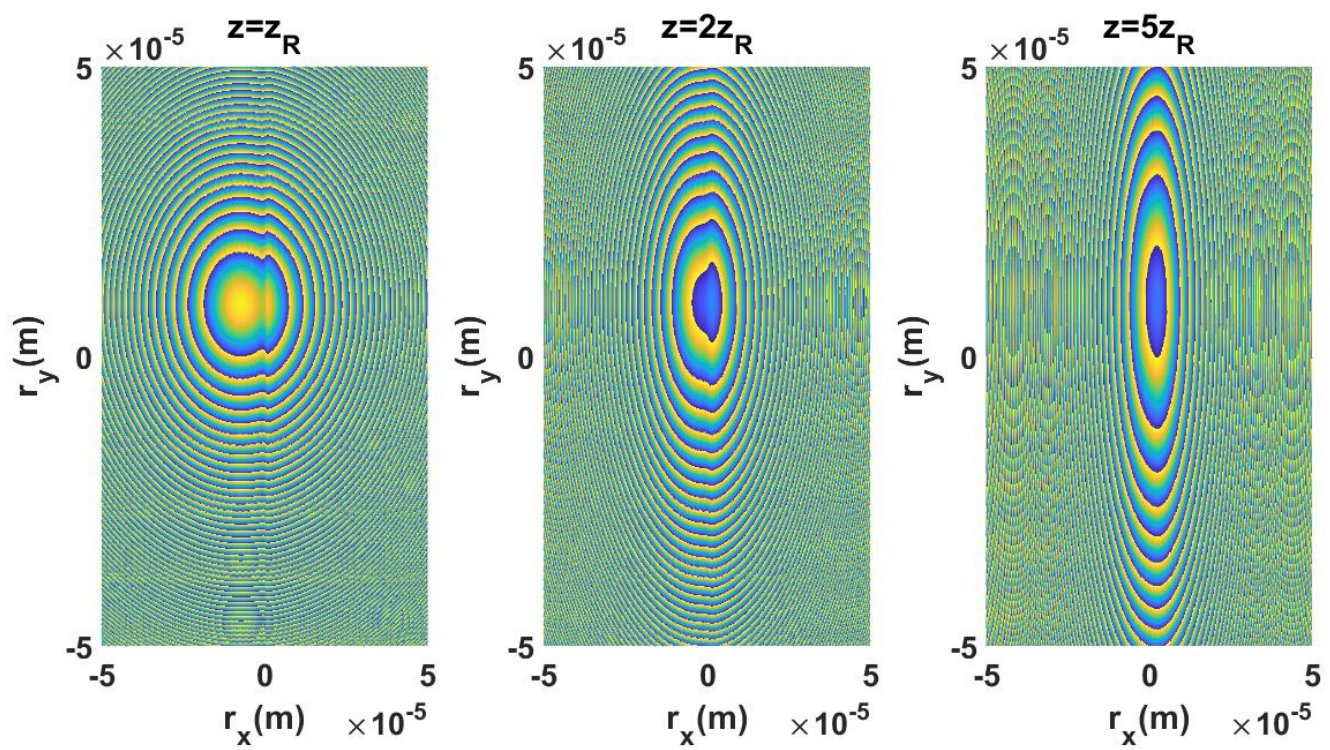

Figure 12. Phase distribution of chirped sinh-Gaussian beam having $\boldsymbol{c}=\mathbf{1}, \boldsymbol{d}=\mathbf{3}$ and $\boldsymbol{\beta}=$ 0.6 in crystal with $\boldsymbol{e}=\mathbf{1 . 7}$. 


\section{References}

[1] Bayraktar M 2020 Propagation of Airyprime beam in uniaxial crystal orthogonal to propagation axis Optik 166183

[2] Xu C J, Lin L D, Huang Z Z, He D L and Deng D M 2019 Propagation of a Pearcey beam in uniaxial crystals Chinese Phys $B \mathbf{2 8}$

[3] Xu C J, Lin L D, Huang Z Z, Chen Y Z, Yang X B, Liu H Z and Deng D M 2018 Propagation of a radially polarized Pearcey beam in uniaxial crystals Laser Phys $\mathbf{2 8}$

[4] Zhou G Q, Chen R P and Chu X X 2012 Propagation of Airy beams in uniaxial crystals orthogonal to the optical axis Opt Express 20 2196-205

[5] Zhou M L, Chen C D, Chen B, Peng X, Peng Y L and Deng D M 2015 Propagation of an Airy-Gaussian beam in uniaxial crystals Chinese Phys $B \mathbf{2 4}$

[6] Yu W H, Zhao R H, Deng F, Huang J Y, Chen C D, Yang X B, Zhao Y P and Deng D M 2016 Propagation of Airy Gaussian vortex beams in uniaxial crystals Chinese Phys $B 25$

[7] Li D D, Peng X, Peng Y L, Zhang L P and Deng D M 2017 Nonparaxial evolution of the Airy-Gaussian vortex beam in uniaxial crystal J Opt Soc Am B 34 891-8

[8] Wang L Y, Zhang J B, Feng L Y, Pang Z H, Zhong T F and Deng D M 2018 Propagation properties of chirped Airy vortex beams with A-polarization through uniaxial crystals Chinese Phys $B \mathbf{2 7}$

[9] Zhang J B, Zhou K Z, Liang J H, Lai Z Y, Yang X L and Deng D M 2018 Nonparaxial propagation of the Chirped Airy vortex beams in uniaxial crystal orthogonal to the optical axis Opt Express 26 1290-304

[10] Chen Y Z, Zhao G W, Ye F, Xu C J and Deng D M 2018 Nonparaxial propagation properties of the chirped Airy Gaussian vortex beams in uniaxial crystals orthogonal to the optical axis Chinese Phys $B 27$

[11] Chen Y H, Wu L X, Mo Z X, Wu L C and Deng D M 2021 Nonparaxial propagation of radially polarized chirped Airy beams in uniaxial crystal orthogonal to the optical axis* Chinese Phys B 30 
[12] Sun C, Lv X, Deng D M, Ma B B, Liu H Z and Hong W Y 2019 Nonparaxial propagation of the radially polarized Airy-Gaussian beams with different initial launch angles in uniaxial crystals Opt Commun 445 147-54

[13] Zhou G Q 2012 Characteristics of paraxial propagation of a super Lorentz-Gauss SLG(01) mode in uniaxial crystal orthogonal to the optical axis Chinese Phys B 21

[14] Xu Y Q, Zhou G Q and Wang X G 2013 Nonparaxial propagation of HermiteLaguerre-Gaussian beams in uniaxial crystal orthogonal to the optical axis Chinese Phys B 22

[15] Ye J R, Zhang J B, Ye F, Xie J T and Deng D M 2020 Propagation properties of the rotating elliptical chirped Gaussian beam in uniaxial crystals orthogonal to the optical axis Wave Random Complex

[16] Bayraktar M 2021 Properties of hyperbolic sinusoidal Gaussian beam propagating through strong atmospheric turbulence Microw Opt Techn Let

[17] Zhang Y L, Zhou X X and Yuan X H 2019 Performance analysis of sinh-Gaussian vortex beams propagation in turbulent atmosphere Opt Commun 440 100-5

[18] Wu H, Dan Y Q, Yu X P, Xu Y G and Deng N 2020 Effect of outer scale on partially coherent Sinh-Gaussian beams in inhomogeneous atmospheric turbulence J Mod Optic 67 890-8

[19] Hricha Z, Yaalou M and Belafhal A 2021 Propagation of hollow sinh-Gaussian beams in strongly nonlocal nonlinear media Opt Commun 478

[20] Liu Z R, Wang X and Hang K L 2019 Enhancement of trapping efficiency by utilizing a hollow sinh-Gaussian beam Sci Rep-Uk 9

[21] Bayraktar M 2021 Average intensity of astigmatic hyperbolic sinusoidal Gaussian beam propagating in oceanic turbulence Phys Scripta 96

[22] Senthilkumar M, Rajesh K B, Udhayakumar M, Jaroszewicz Z and Mahadevan G 2019 Focusing properties of spirally polarized sinh Gaussian beam Opt Laser Technol $111623-8$

[23] Born M and Wolf E 1999 Principles of optics (Oxford: Pergamon)

[24] Yariv A and Yeh P 1984 Optical waves in crystals (New York: Wiley)

[25] Gradshteyn I S and Ryzhik I M 2015 Table of Integrals, Series, and Products: Academic Press) 
Figures

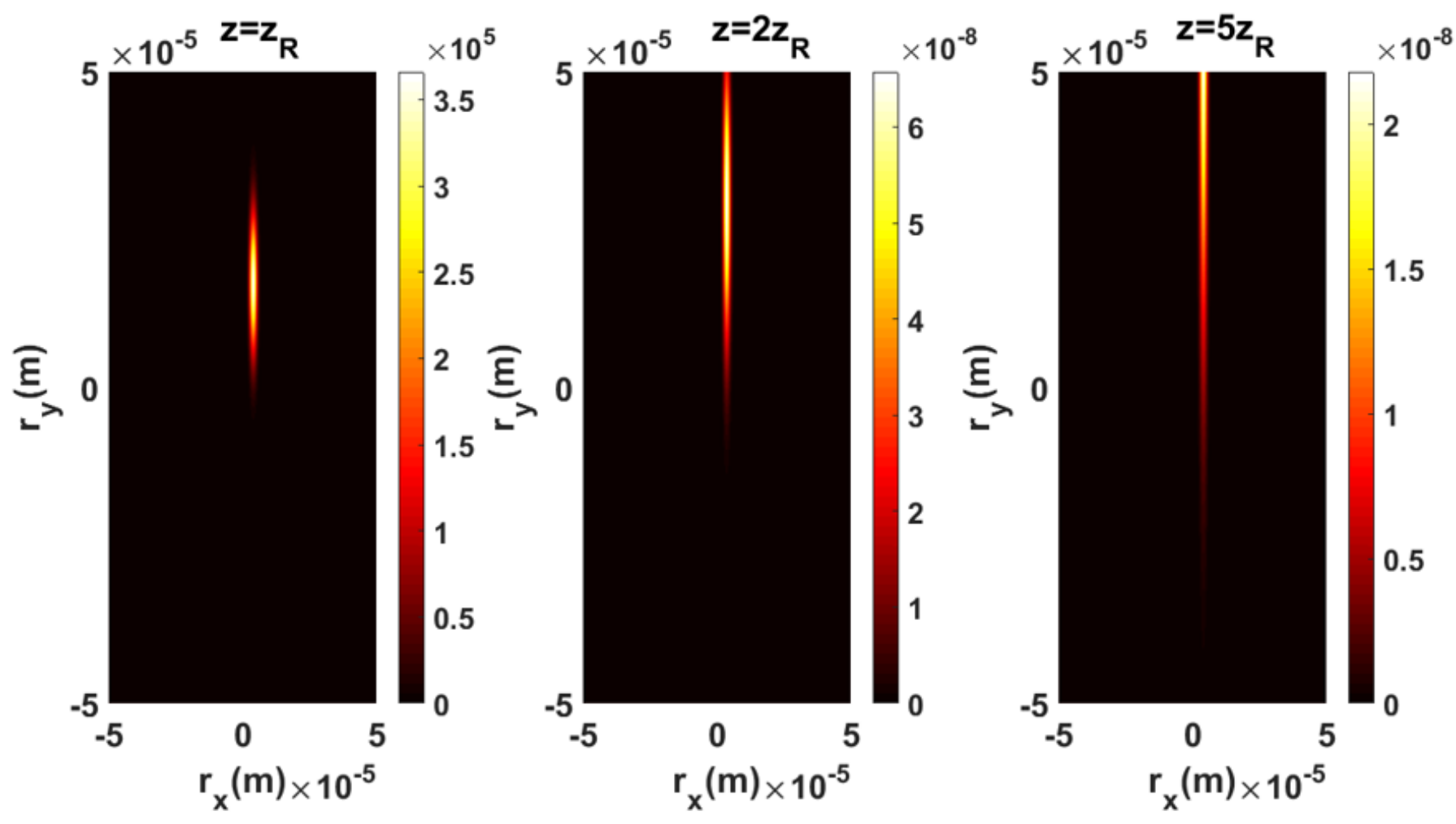

Figure 1

Intensity distribution of chirped sinh-Gaussian beam having $c=d=1$ and $\beta=0.6$ in crystal with $e=0.7$. 


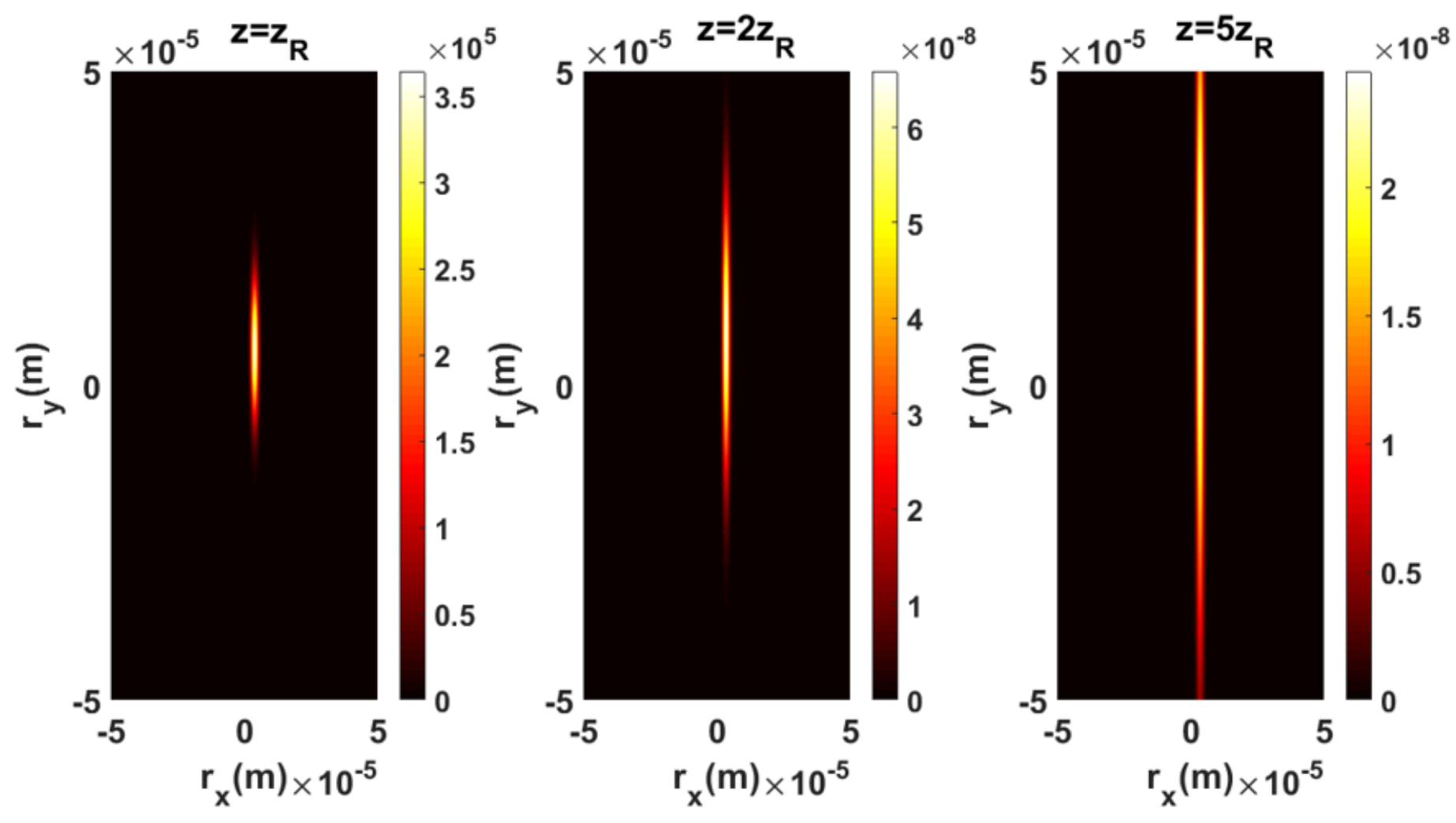

Figure 2

Intensity distribution of chirped sinh-Gaussian beam having $c=d=1$ and $\beta=0.1$ in crystal with $e=0.7$.

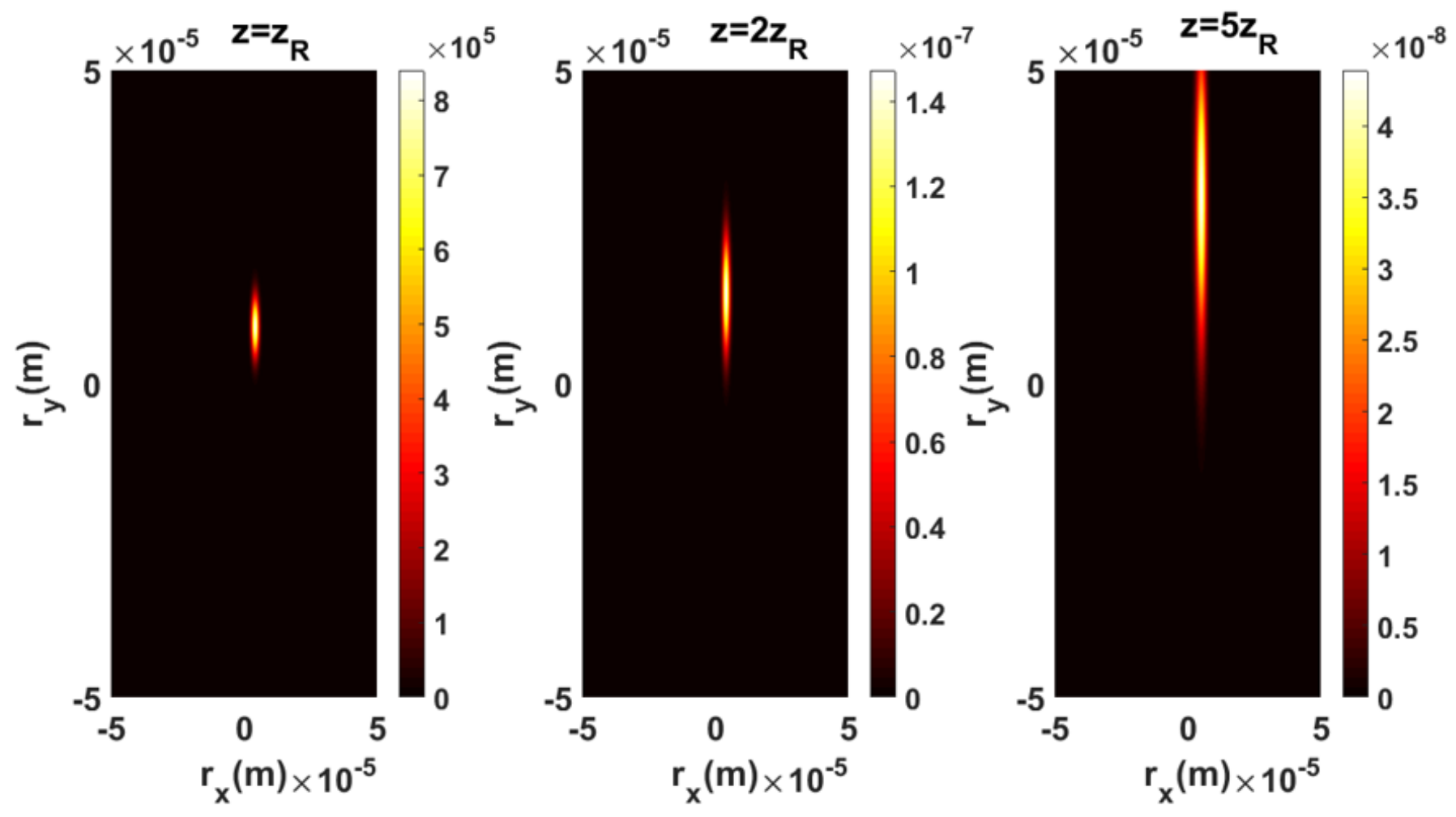


Figure 3

Intensity distribution of chirped sinh-Gaussian beam having $c=d=1$ and $\beta=0.6$ in crystal with $e=1.7$.

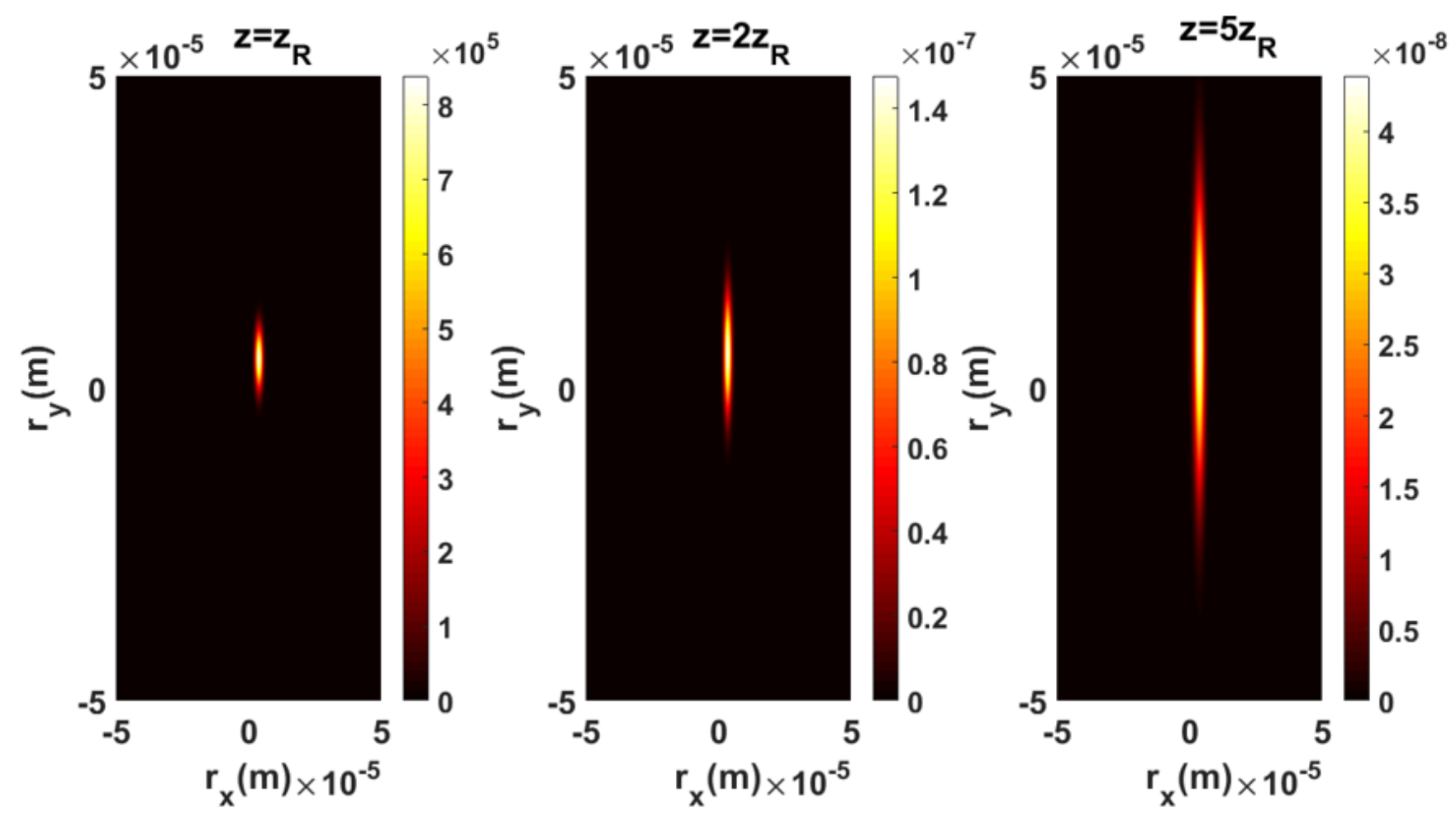

Figure 4

Intensity distribution of chirped sinh-Gaussian beam having $c=d=1$ and $\beta=0.1$ in crystal with $e=1.7$. 


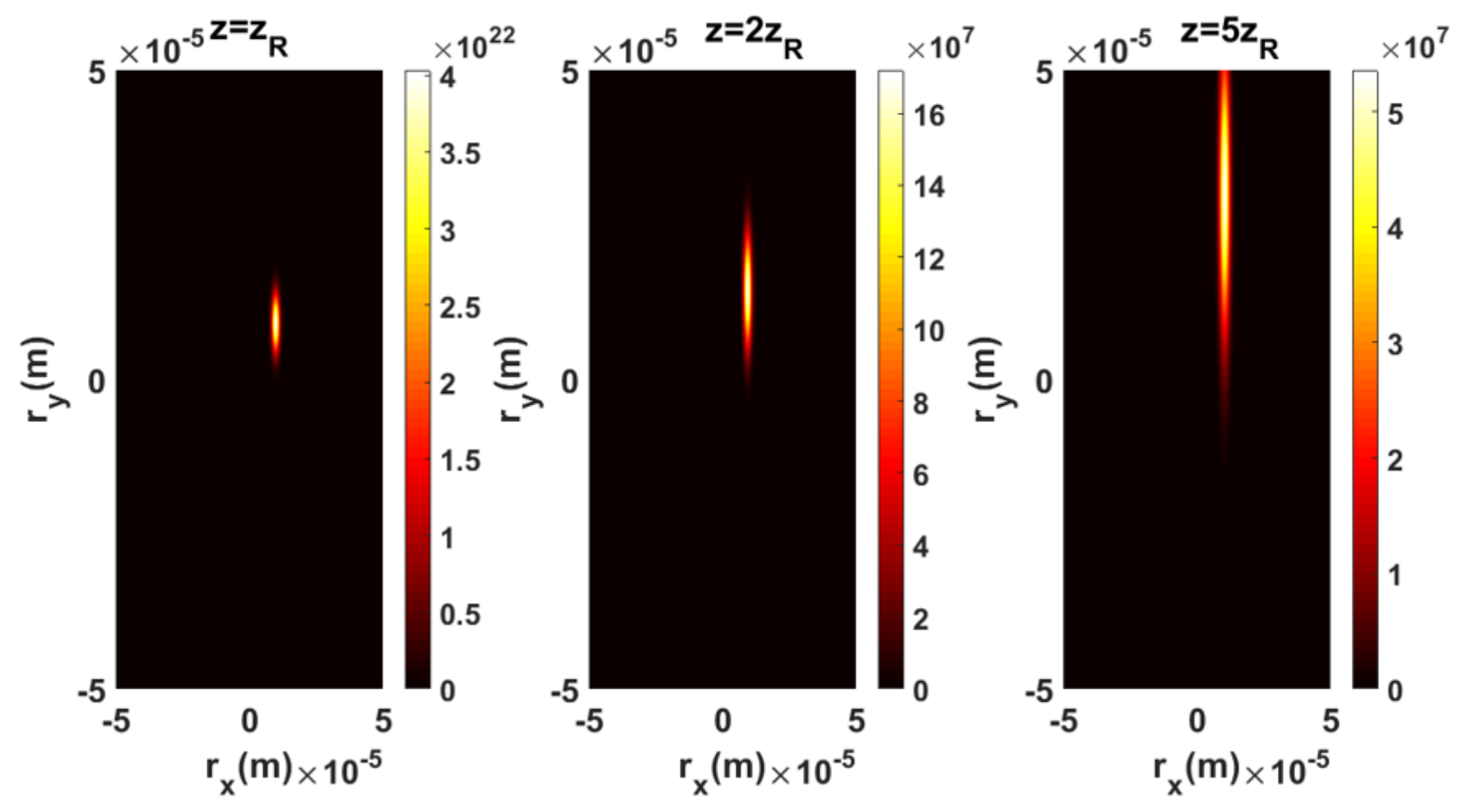

Figure 5

Intensity distribution of chirped sinh-Gaussian beam having $c=3, d=1$ and $\beta=0.6$ in crystal with $e=1.7$.

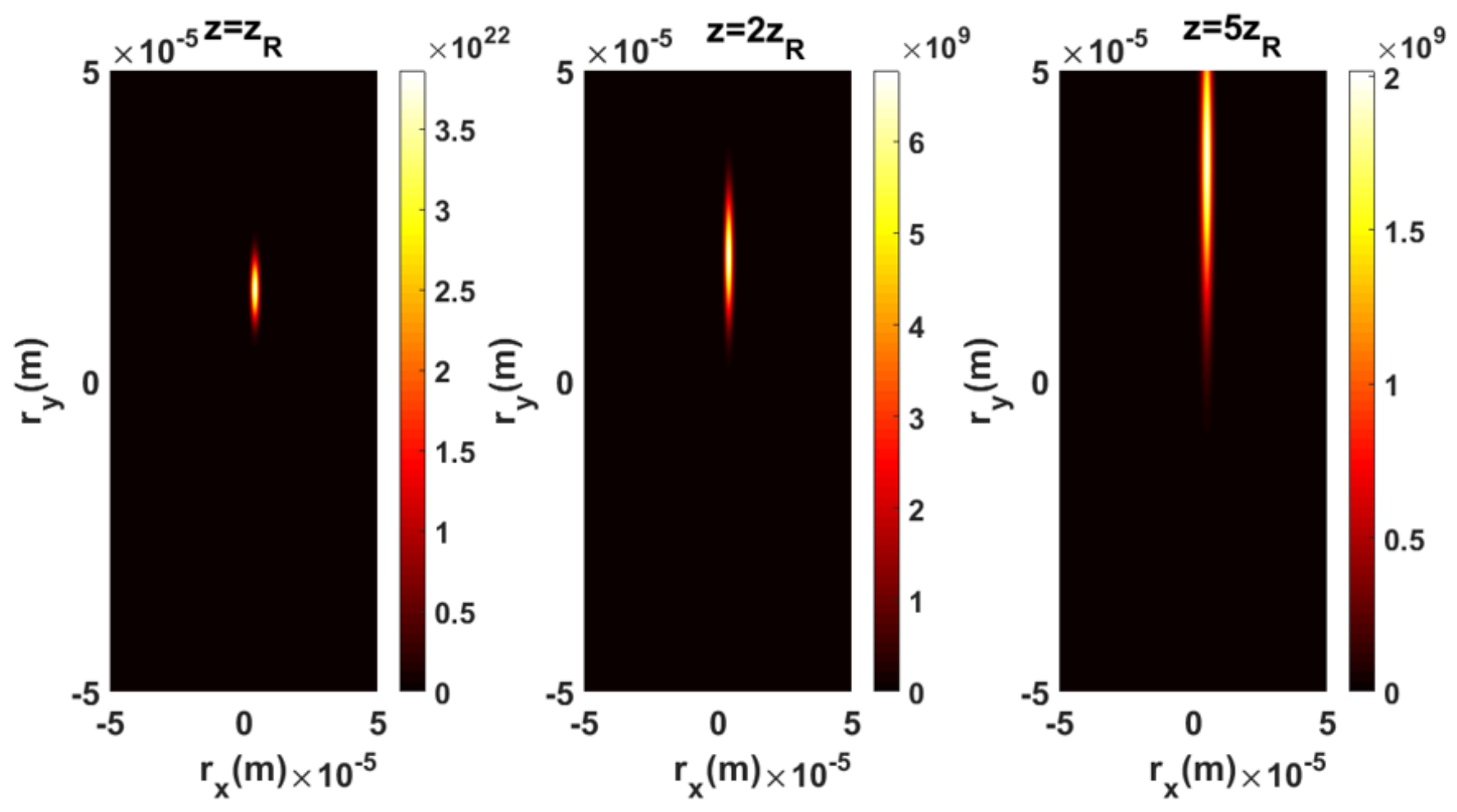

Figure 6 
Intensity distribution of chirped sinh-Gaussian beam having $c=1, d=3$ and $\beta=0.6$ in crystal with $e=1.7$.
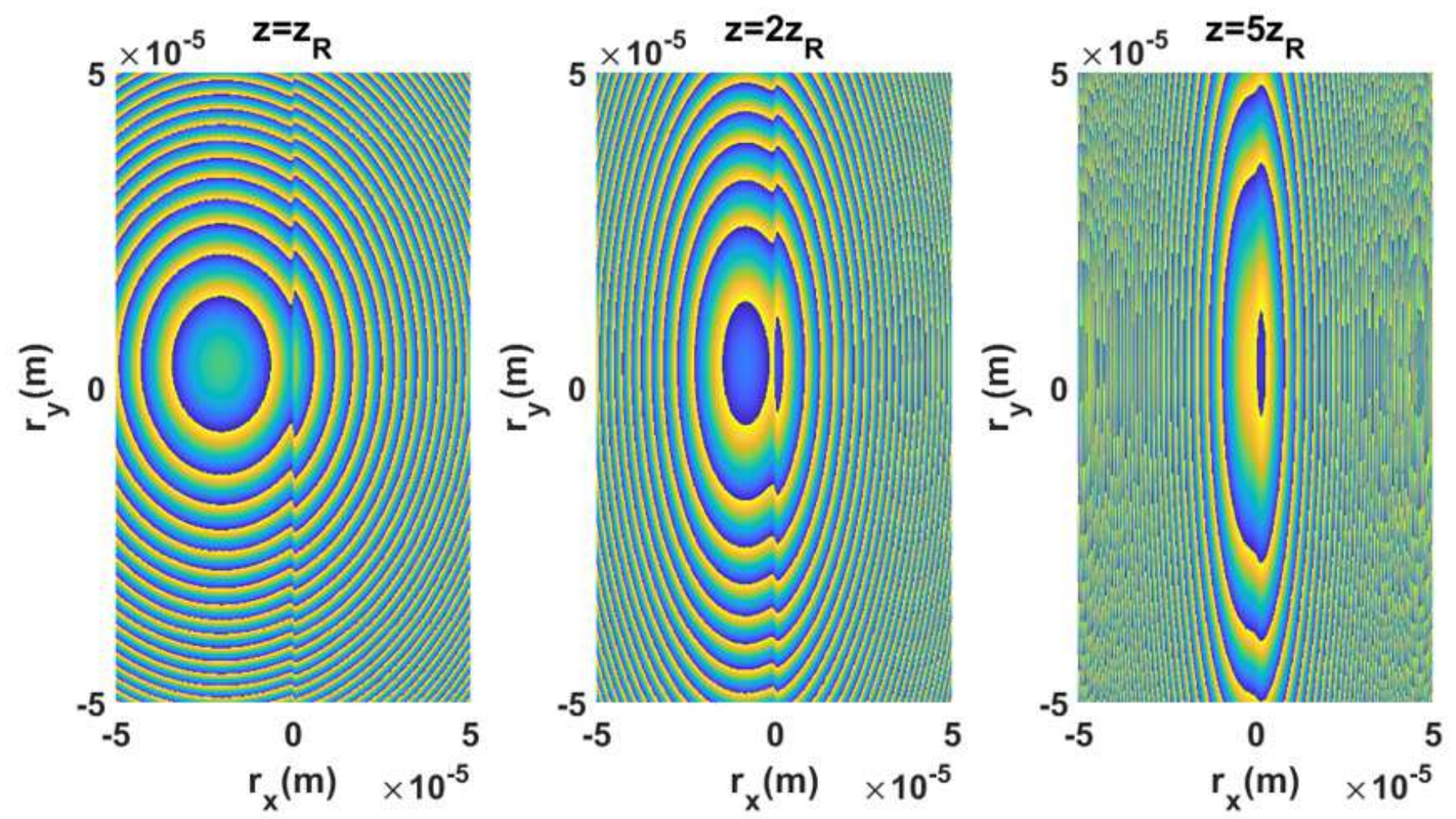

Figure 7

Phase distribution of chirped sinh-Gaussian beam having $c=d=1$ and $\beta=0.6$ in crystal with $e=0.7$.
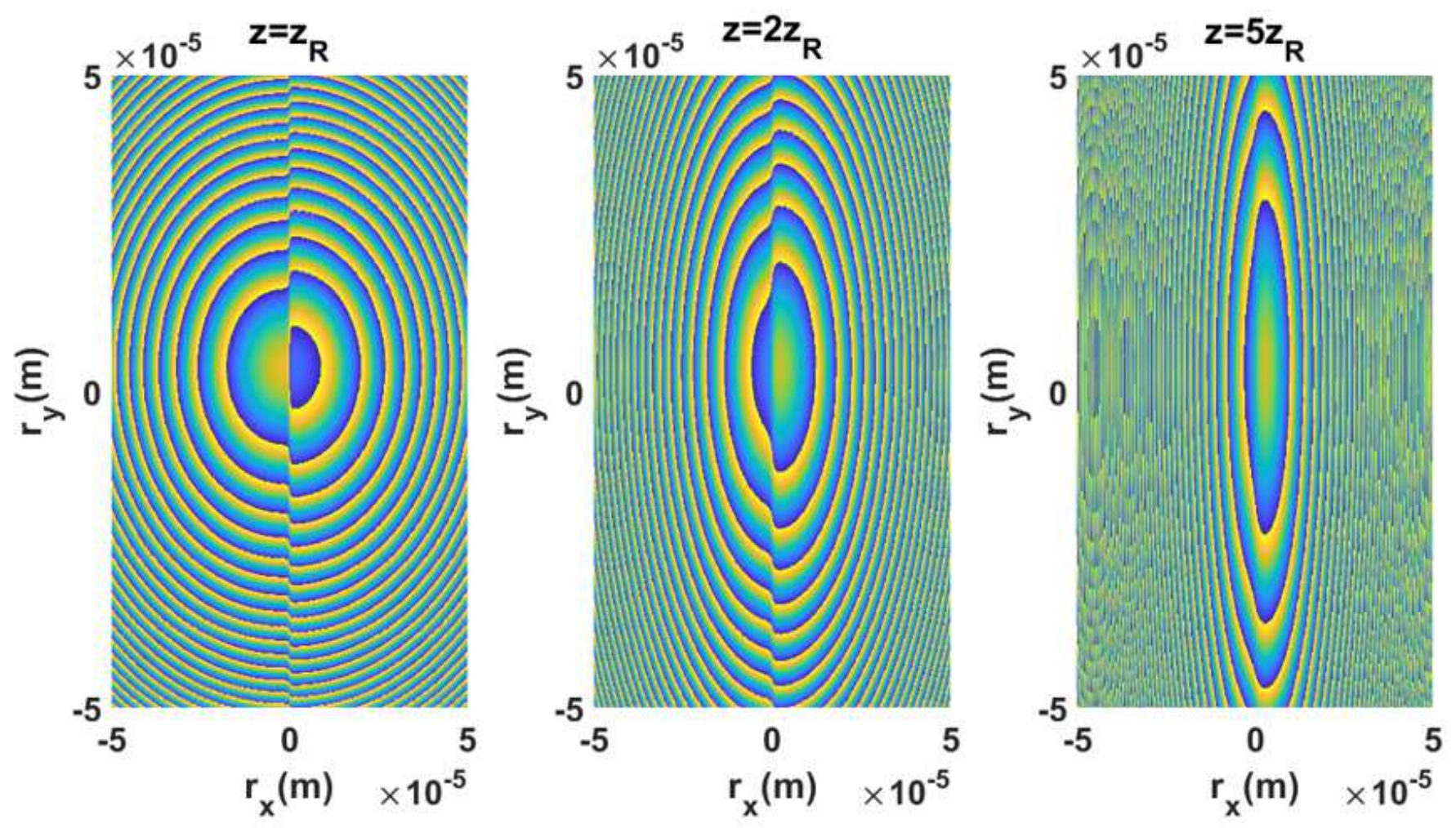
Figure 8

Phase distribution of chirped sinh-Gaussian beam having $c=d=1$ and $\beta=0.1$ in crystal with $e=0.7$.
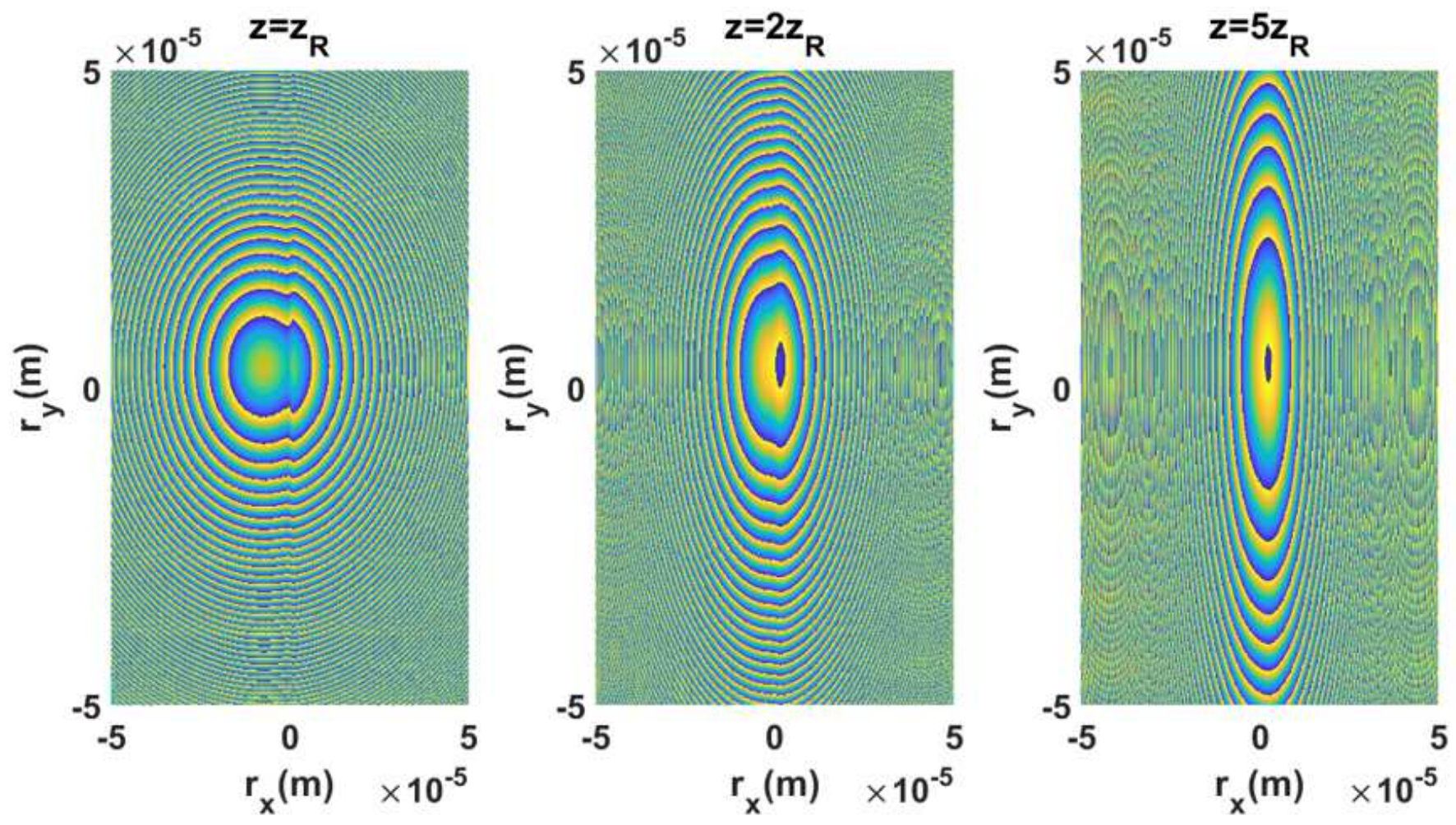

Figure 9

Phase distribution of chirped sinh-Gaussian beam having $c=d=1$ and $\beta=0.6$ in crystal with $e=1.7$. 

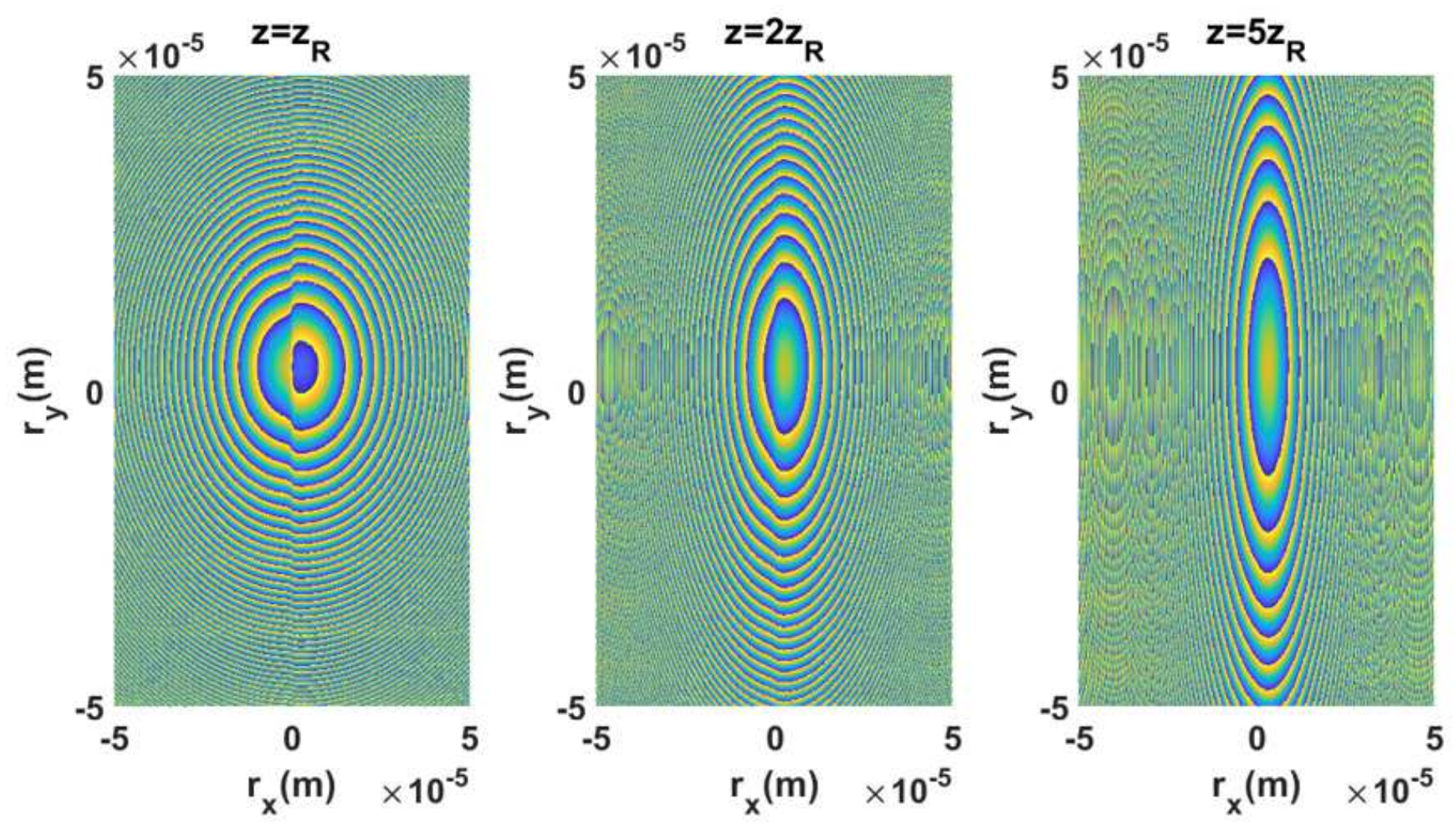

Figure 10

Phase distribution of chirped sinh-Gaussian beam having $c=d=1$ and $\beta=0.1$ in crystal with $e=1.7$.
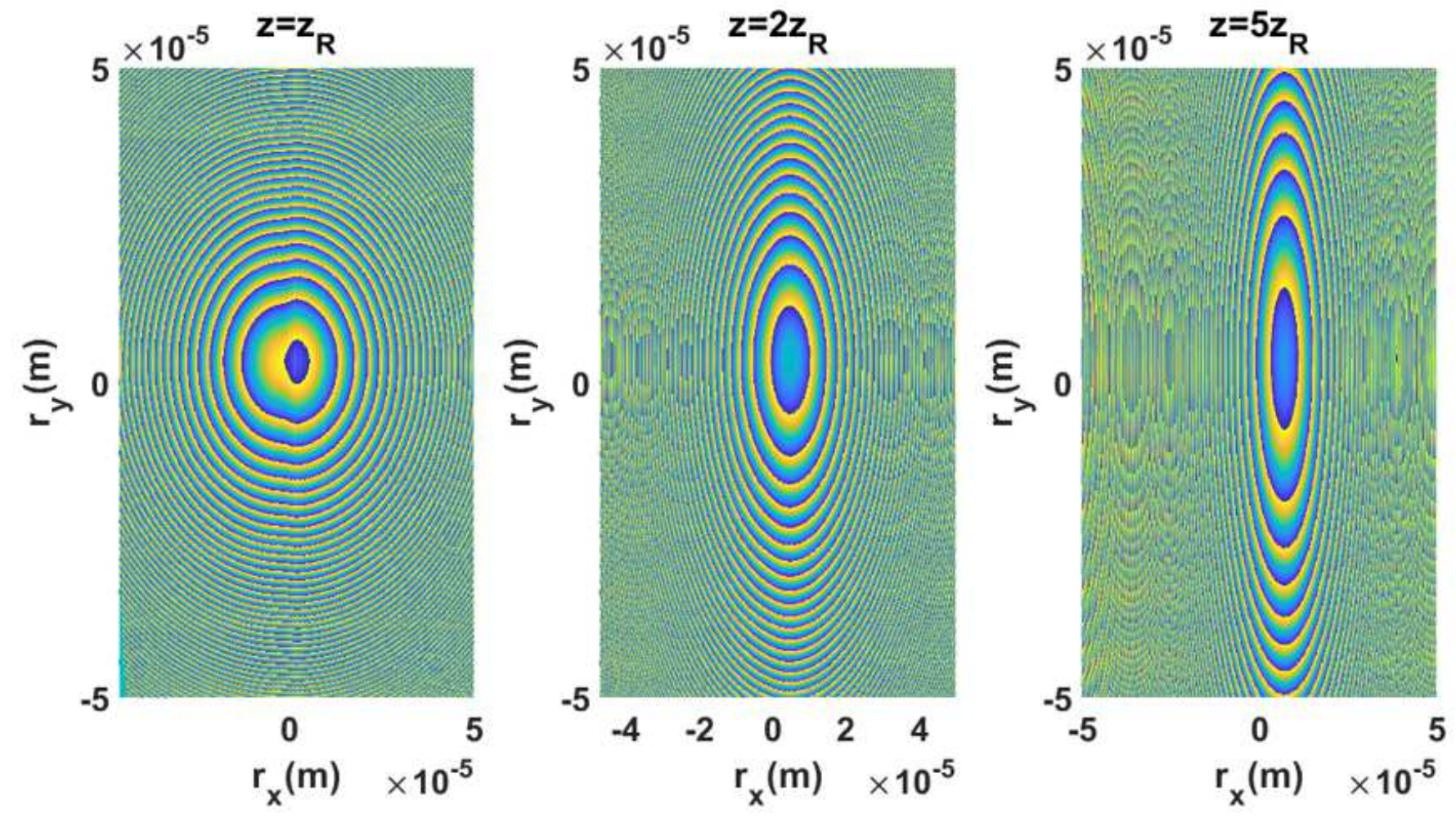

Figure 11 
Phase distribution of chirped sinh-Gaussian beam having $c=3, d=1$ and $\beta=0.6$ in crystal with $e=1.7$.
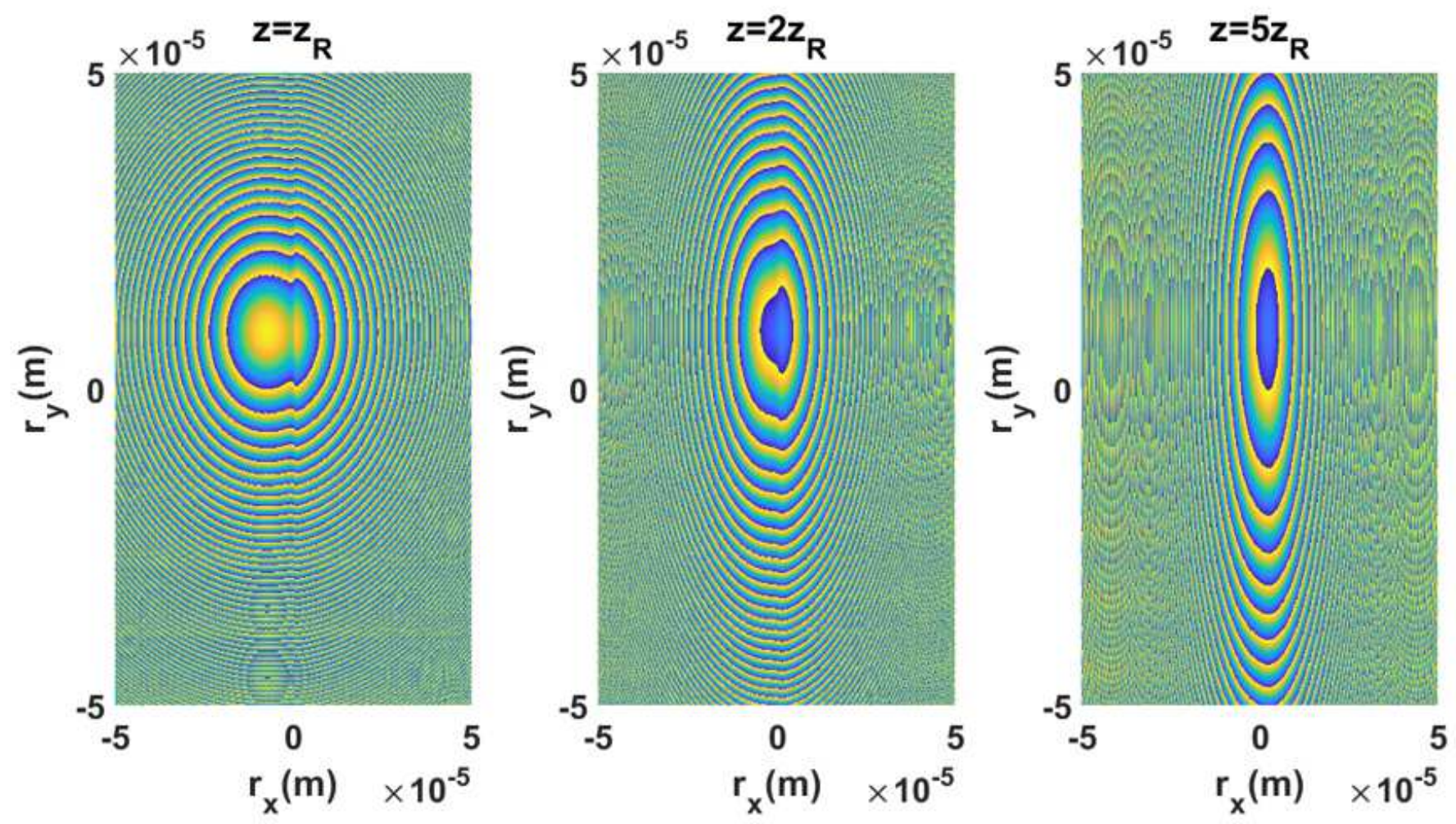

Figure 12

Phase distribution of chirped sinh-Gaussian beam having $c=1, d=3$ and $\beta=0.6$ in crystal with $e=1.7$. 